\title{
2014 startet nationale Kohorte mit starker radiologischer Beteiligung
}

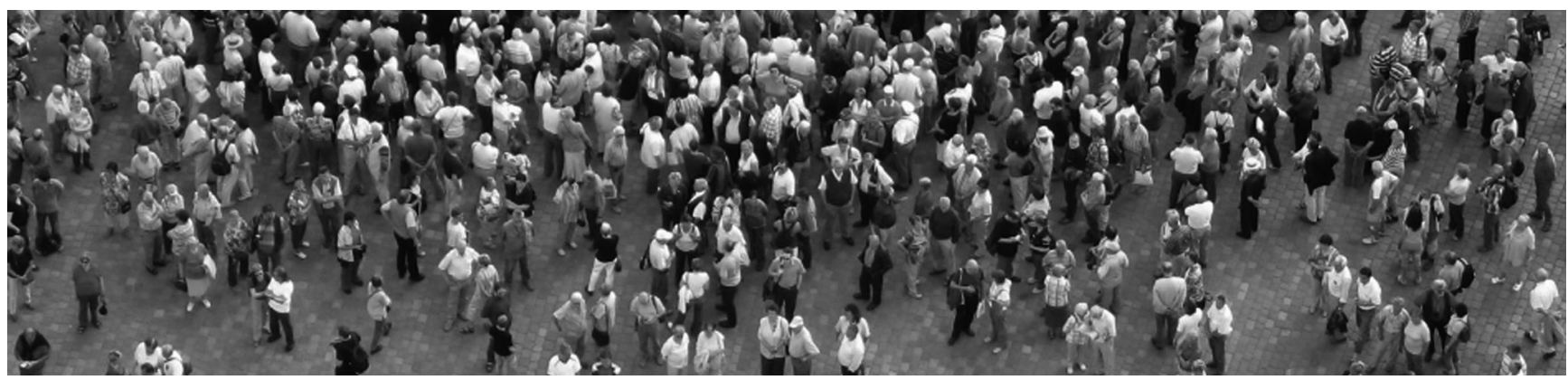

Berlin, 15. Januar 2014. Im Frühjahr 2014 läuft die bislang größte bundesweite Langzeitstudie „Gemeinsam forschen für eine gesündere Zukunft - Die Nationale Kohorte“ (NAKO) an. Die Radiologie spielt hier eine wesentliche Rolle: Von den insgesamt 200000 Personen, die die NAKO innerhalb der nächsten 30 Jahre untersucht, erhalten rund 30000 Probanden auch eine Ganzkörper-MRT.

Die Studie hat zum Ziel, die Entstehung von chronischen Erkrankungen näher zu erforschen und dabei auch den Zusammenhang mit Umweltfaktoren und dem Lebensstil der Studienteilnehmer einzubeziehen. Die NAKO wird außerdem Krankheitsrisikofaktoren und Biomarker für die Früherkennung von Krankheiten identifizieren und effektive Präventionsstrategien entwickeln. Hintergrund des Forschungsprojekts ist die Zunahme chronischer Erkrankungen wie Krebs, Diabetes mellitus und Herz-Kreislauf-Erkrankungen, die als Folge des demografischen Wandels zu erwarten ist.
Ein wesentlicher Unterschied der Nationalen Kohorte gegenüber anderen Studien in Deutschland, Europa und den USA, wie etwa der Heinz Nixdorf Recall-Studie in Deutschland, der Rotterdam-Studie in den Niederlanden oder MESA in den USA, liegt in der landesweiten Orientierung der Studie und der herausgehobenen Bedeutung der wissenschaftlichen Radiologie: Etwa 30000 Probanden erhalten zusätzlich zur Basis- auch eine GanzkörperMRT-Untersuchung. Der MRT-Teil der Studie, der an 5 Studienzentren in Deutschland durchgeführt wird, wird von einem interdisziplinären wissenschaftlichen Konsortium unter der Leitung von PD Dr. Fabian Bamberg aus München koordiniert.

„Die Nationale Kohorte wird die wissenschaftliche Seite der Radiologie in Deutschland erheblich stärken“, sagt Professor Norbert Hosten, Präsident der Deutschen Röntgengesellschaft. Dr. Bamberg fügt hinzu: „Bis jetzt ist OutcomeForschung in der Radiologie schwer durchzuführen. Sie wird aber von Kostenträgern und auch vom Gemeinsamen Bundesausschuss gefordert, um den Nutzen neuer Bildgebungs-Technologien nachzuweisen. Deswegen freuen wir uns, wenn die Radiologie wesentlich zu den Ergebnissen der NAKO beitragen und die Outcome-Forschung davon profitieren kann. So wird die Radiologie künftig auch neue Risikomarker in der MRT etablieren können.“

Die Nationale Kohorte wird vom Bundesministerium für Bildung und Forschung, den 14 beteiligten Bundesländern und der Helmholtz-Gemeinschaft Deutscher Forschungszentren mit insgesamt 210 Millionen $€$ finanziert. Davon entfallen rund 21 Millionen $€$ auf den radiologischen Teil der Studie.

Weitere Informationen: www.nationale-kohorte.de www.bmbf.de/press/3480.php

Anne-Katrin Hennig, Pressestelle der DRG 